\title{
BMJ Open Resilience support to enhance positive health outcomes for police officers in five Anglosphere nations: a scoping review protocol
}

\author{
Audrey Steenbeek (D , ${ }^{1,2}$ Chris Giacomantonio, ${ }^{3}$ Arlene Brooks, ${ }^{3}$ Camilla Holmvall, ${ }^{4}$ \\ Ziwa Yu, ${ }^{1,2}$ Melissa Rothfus ${ }^{5}$
}

To cite: Steenbeek A, Giacomantonio C, Brooks A, et al. Resilience support to enhance positive health outcomes for police officers in five Anglosphere nations: a scoping review protocol. BMJ Open 2020;10:e038895. doi:10.1136/ bmjopen-2020-038895

- Prepublication history and additional material for this paper is available online. To view these files, please visit the journal online (http://dx.doi.org/10. 1136/bmjopen-2020-038895)

Received 27 March 2020 Revised 19 0ctober 2020 Accepted 28 0ctober 2020

Check for updates

(C) Author(s) (or their employer(s)) 2020. Re-use permitted under CC BY-NC. No commercial re-use. See rights and permissions. Published by BMJ.

${ }^{1}$ Nursing, Dalhousie University, Halifax, Nova Scotia, Canada ${ }^{2}$ Aligning Health Needs and Evidence for Transformative Change (AH-NET-C): A Joanna Briggs Institute Centre of Excellence, School of Nursing, Dalhousie University, Halifax, Nova Scotia, Canada

${ }^{3}$ Halifax Regional Police, Halifax, Nova Scotia, Canada

${ }^{4}$ Psychology \& Management, Saint Mary's University, Halifax, Nova Scotia, Canada

${ }^{5}$ W.K. Kellogg Health Sciences Library, Dalhousie University, Halifax, Nova Scotia, Canada

Correspondence to

Dr Audrey Steenbeek; a.steenbeek@dal.ca

\section{ABSTRACT}

Introduction Law enforcement involves exposure to threatening situations and traumatic events that place police officers at risk for negative physical and mental health outcomes. Resilience support, among other elements of training, may help mitigate these risks, yet little is known about which aspects of resilience support help officers achieve better health and quality of life outcomes.

Methods and analysis This review will consider all literature that examines the links between resilience support, physical/mental health and quality of life outcomes for police officers in five Anglosphere nations: Canada, the USA, Australia, New Zealand and the UK. This review will include all literature (including those that show null or negative links) involving any public policing agency that has a formal rank structure and includes a localized, uniformed emergency response function. Resilience support may include, but is not limited to: tools, policies, models, frameworks, programmes and organizational features that seek to promote positive, physical/mental health and quality of life outcomes at three levels of resilience: (1) readiness and preparedness, (2) response and adaptation, (3) recovery and adjustment. Peer reviewed and grey literature examining resilience support since 2000 that focuses on police officers are eligible for inclusion. Databases/sources to be searched will include: PsycINFO, Academic Search Premier, CINAHL, Public Affair Index, Campbell Collaboration, ProQuest Dissertations and Theses Global, Business Source Complete, Scopus and Google. Retrieval of full-text, English-language studies (and other literature), data extraction, data synthesis and data mapping will be performed independently by two reviewers, following Joanna Briggs Institute methodology. Ethics and dissemination Ethics approval is not required for this scoping review, and the literature search will start in November 2020 or upon acceptance of this protocol. The findings of the scoping review will be available [April 2021] and will be published in a peer reviewed journal.

\section{INTRODUCTION}

Among first responders, police officers are often exposed to dangerous situations. ${ }^{1} 2$ Police officers are also subjected to a variety of additional stressful conditions that include
Strengths and limitations of this study

- The proposed scoping review will provide knowledge around resilience support for police officers in five Anglosphere nations.

- The proposed scoping review will identify and map peer-reviewed publications and grey literature (e.g. government reports), though some literature may be missed.

- Rigorous screening/data extraction will be done by two independent reviewers; however, articles in the final review will not be evaluated for methodological quality.

- The search is limited to English-language literature published in 2000 onwards.

- The review may only be applicable to police officers/ organizations in Anglosphere nations.

internal (organizational) stress, such as excessive reporting, ${ }^{1}$ limited career mobility, ${ }^{2}$ rotating shifts, ${ }^{2}$ long overtime hours, ${ }^{2}$ insufficient training, ${ }^{12}$ rigid bureaucratic policies ${ }^{3}$ and external stressors such as those associated with daily interactions with the criminal justice system and the general public. ${ }^{4}$ Officers also experience task-focused stressors such as those related to the use of discretion ${ }^{4}$ and spontaneous decision making in ambiguous situations. ${ }^{1}$ Equally concerning, is the repeated exposure to potentially traumatic situations (i.e., critical incidents) such as armed confrontations, motor-vehicle crashes and witnessing violent deaths, among others. ${ }^{5}$

Frequent exposure to these stressors place police officers at risk of experiencing poor physical and mental health outcomes that may include: depression, ${ }^{1}$ post-traumatic stress disorder, ${ }^{1} 26$ impaired psychosocial functioning, ${ }^{6}$ substance abuse, ${ }^{16}$ increased risk of suicidal behaviour, ${ }^{16}$ cardiovascular disease, ${ }^{1}{ }^{6} 7$ hypervigilance, ${ }^{6}$ metabolic syndrome, ${ }^{78}$ sleep disorders ${ }^{78}$ and increased 
susceptibility to common colds or influenza, ${ }^{1}$ among many other complaints. These stressors can also be detrimental to the law-enforcement organization. ${ }^{89}$ Law-enforcement stressors may result in increased aggression among officers, ${ }^{10}$ greater absenteeism ${ }^{10}$ as well as lower job performance and satisfaction ${ }^{10}$ and reduced efficiency. ${ }^{9}$ Officers working under high-stress conditions often experience strain and occupational burnout that may result in minimal involvement with others in the organization and reduced commitment to completing assigned tasks. ${ }^{1}$

As is the case with other first responders (e.g., paramedics, firefighters), police officers are obliged to respond to traumatic and stressful events in the course of their occupational duties. ${ }^{11}$ Trauma is not a new phenomenon, and its effects have long been studied in the military context. ${ }^{12}$ Chapin et al (p83) ${ }^{13}$ further add ' $\ldots$ although police work may not generate the same intensity of stress as military combat, the cumulative effects of numerous traumatic exposures layered on top of the routine bureaucratic and administrative stressors can have powerful effects on police.' Essentially, exposure to trauma is an inherent part of law enforcement work, and for this reason, officers and policing organizations may misunderstand or underplay the effects of trauma, ${ }^{11}$ and officers may resist seeking help. ${ }^{1}$ In the law enforcement subculture, the idea of seeking a mental-health or stress-reduction intervention has traditionally been viewed unfavourably, with those who use these services often labelled as weak or non-resilient. ${ }^{1}$ In an onlinesurvey study of 421 constables from a large municipal police force in Canada, Heffren et al found that 102 officers reported experiencing traumatic events, of whom, $44.1 \%$ attempted to deal with the situation themselves with only $17.6 \%$ seeking professional help. ${ }^{14}$ According to Blum, this lack of health-seeking behaviour may be due in part to the stigma associated with mental illness and a perceived lack of anonymity when seeking mental-health assistance in matters involving critical incident stress. ${ }^{15}$

Similar to the principles of crime prevention, the key to physical and mental well-being may also be found in prevention, through a range of structures, interventions and services that we may broadly conceive of as resilience support. ${ }^{11}$ If resilience support (broadly conceived) is routinely implemented, police officers, trainers and cadets may consider it to be a natural component of police organizational processes and accept it as part of being an officer. ${ }^{11} 16$ Additionally, considering a broader view of health and quality of life outcomes, which includes accessing services and supports to target well-being, resilience support may also be beneficial in preventing mental illness from occurring and/or improve health seeking behaviours among police officers. According to Ungar, resilience is the capacity of an individual (or system) to anticipate, adapt and reorganize itself successfully to disturbances that threaten system function, viability or development. The concept can be applied to many kinds of individual and community systems, and at several interacting levels. ${ }^{17-21}$
Resilience support has the potential to improve an officer's well-being by increasing the capacity of an individual and/or an organization to anticipate (i.e., readiness and preparedness), adapt (i.e., response and adaption) and grow (i.e., recovery and adjustment) in the face of adversity, acute crises and chronic stress; it is a process that requires a multifaced, interdisciplinary strategy and a systems review. ${ }^{22}$ Resilience support has the ability to help individuals identify their own strengths and barriers as well as broader environmental and organizational factors that are most amenable to change; research suggests that innovation, ingenuity, creativity and novelty, among other factors, are critical for resilience support to be effective, and organizations and governance structures are critical in promoting and enabling these processes. ${ }^{22}$ Increasing resilience has been seen as an effective tool to help police officers and organizations deal with the many stressors associated with their occupation, ${ }^{16}$ yet literature describing all available resilience support aimed at achieving positive physical/mental health outcomes for police officers has not been comprehensively reviewed and synthesized. While there are several, narrower, systematic reviews in this area, ${ }^{23-25}$ a scoping review that focuses on a broader scope of resilience-support literature (i.e., including peer reviewed and grey literature) would be valuable for policing organizations by mapping existing evidence (including evidence that shows null or negative links) and where possible, highlighting the level of efficacy of different forms of resilience support for police officers. A preliminary search was conducted in Joanna Briggs Institute (JBI) Database of Systematic Reviews/Implementation Reports, Cochrane Library, PROSPERO, CINAHL and MEDLINE on 10 March 2020, no scoping reviews (completed or underway) were identified on this topic.

To identify and map all available evidence around resilience support for police officers, the proposed scoping review will: identify knowledge gaps, clarify key concepts and examine all forms of literature that explores various types of resilience support and associated health and quality of life outcomes for police officers in Canada, the USA, Australia, New Zealand and the UK. The authors also aim to explore several key characteristics associated with resilience support such as tools, strategies, policies, frameworks, programmes and organizational features, among others, associated with health and quality of life outcomes through three levels of resilience: (1) readiness and preparedness, (2) response and adaptation, (3) recovery and adjustment. ${ }^{22}{ }^{26}$ Additionally, the results from this review will be used towards the development of a future systematic review on the same subject area.

\section{REVIEW QUESTIONS}

What is the available evidence around links between resilience support and health and quality of life outcomes for police officers in Canada, the USA, Australia, New Zealand and the UK? 
Subquestions: What tools, strategies, training, organizational features, policies, frameworks and programmes are available? What are their key characteristics and potential outcomes (i.e., positive, null, negative)?

\section{METHODS AND ANALYSIS Eligibility criteria \\ Participants}

This scoping review will consider all literature involving any public policing agency that has a formal rank structure and includes as part of its mandate a localized, uniformed emergency response function. This will include local, municipal, regional and federal law enforcement officers (eg, Royal Canadian Mounted Police (RCMP) in Canada) from five Anglosphere nations. Retired police officers, military police and border patrol officers will be excluded.

\section{Concept}

All literature, including those that show null or negative associations, will be considered if it examines links between resilience support and physical/mental health and quality of life outcomes for police officers and/or policing organizations. While there are several definitions of resilience, for the purpose of this scoping review, it pertains to the capacity of an individual (or system) to anticipate, adapt and reorganize itself successfully to disturbances that threaten system function, viability or development. ${ }^{22}{ }^{26}$ Resilience support may include, for example, tools, strategies, training, policies, frameworks, programmes and organizational features that seek to enhance positive health and quality of life outcomes by increasing the capacity of an individual and/or an organization to anticipate (i.e., readiness and preparedness), adapt (i.e., response and adaption) and grow (i.e., recovery and adjustment). ${ }^{22}{ }^{26}$ Key characteristics of each resilience support identified will also include: guiding principles (e.g., assumptions, values and beliefs), main objectives, intended outcomes and logistical requirements (e.g., human resources, financial, space, time). Any literature that provides narrative text on these characteristics and is linked to health and quality of life outcomes will be considered for inclusion.

\section{Context}

This review will consider all literature that focuses on resilience support delivered in all police settings among active law enforcement personnel, including local, provincial/ state and national/federal personnel at all ranks. This includes civilian roles and contains as part of its mandate a localized, uniformed emergency crime response function. This would include: police personnel, law enforcement personnel, RCMP, highway patrol, state patrol, state trooper, sheriff, Texas ranger, state traffic officer, constabulary, constable, inspector, sergeant, corporal, detective and special constable from the following Anglosphere nations: Canada, the USA, Australia, New Zealand and the UK. These five nations share a common culture and historical ties to the UK, and have similar law enforcement operations, organizations and structures. ${ }^{27}$

\section{Types of literature}

This scoping review will consider any paper that conveys empirical evidence on the effectiveness, efficacy, feasibility and/or appraisal of strategies that address the three levels of resilience: readiness and preparedness, response and adaptation and recovery and adjustment on the physical/ mental health and well-being of police officers. ${ }^{22} 26$ This will include experimental/observational, quantitative/ qualitative and review study designs. Text and opinion papers, conference proceedings, newsletters, theses and dissertations and publicly-available publications by the respective governments of Canada, the USA, Australia, New Zealand and the UK will also be considered for this scoping review if they meet the inclusion criteria. ${ }^{28}$

Published academic and grey literature written in English, and in the year 2000 and beyond will be considered for inclusion. While to some degree an arbitrary dividing line, police organizations in the 1990s and earlier were generally different workplaces than they have been in the 21st century in terms of workforce structure; costs of policing; enhanced use of civilian professionals and crime analysis; changes in the operational environment; increased attention to and recognition of mental health issues; increased gender diversity in policing and so on. ${ }^{29}{ }^{30}$ In turn, studies of officer resilience prior to 2000 may have limited applicability to police services and personnel today. We anticipate that any relevant information or studies that predate 2000 will be incorporated into later literature and consequently, we do not expect to lose relevant information by restricting the search parameters. ${ }^{29} 30$

\section{Information sources}

The databases to be searched will include: PsycINFO, Academic Search Premier, CINAHL, Public Affair Index and Campbell Collaboration. Additionally, Google, ProQuest Dissertations \& Theses Global, Business Source Complete, and Scopus will be searched for grey literature. Hand searches for publicly available grey literature will also be conducted in police government organization websites with each respective country.

\section{Search strategy}

The proposed scoping review will be conducted in accordance with the JBI methodology for scoping reviews. ${ }^{31}$ The search strategy will consist of a comprehensive approach to locate both published and unpublished studies and other literature per inclusion criteria. Following consultation, a qualified health-science librarian developed a search strategy in PsycINFO to identify articles on the topic (see online supplemental appendix 1). This search strategy has been peer reviewed by a second qualified librarian using the Peer Review of Electronic Search Strategies (PRESS) guidelines ${ }^{32}$ and then translated for all 
included information sources. PRESS is a peer reviewed, structured tool, that enhances the quality and comprehensiveness of electronic literature search strategies for both peer reviewed and grey literature. ${ }^{32}$ In addition, the reference lists of all identified articles selected for the scoping review will be screened for additional literature. Key journals related to resilience support for police officers will be hand searched for eligible studies. All literature published in English and from 2000 and onwards will be included to account for the difference in police organization structure.

\section{STUDY RECORDS}

\section{Data management}

Following a comprehensive search of all selected databases, all identified bibliographic citations from each will be uploaded into an online scoping review platform (Covidence) ${ }^{33}$ with duplicates across all the databases removed. Any disagreements that arise between the reviewers at each stage of the study selection process will be resolved through discussion, or with a third reviewer if necessary; Covidence ${ }^{33}$ automates the record keeping processes across the stages of study selection. We will be using the PRISMA-ScR ${ }^{34}$ checklist for scoping reviews that omits discussion of items not relevant to scoping reviews, including: (1) outcomes, (2) priorities, (3) risk of bias in individual studies, (4) meta-bias and (5) confidence in cumulative evidence. The results of the search will be reported in full, and in keeping with the PRISMA extension for scoping reviews-PRISMA-ScR. ${ }^{34}$

\section{Selection process}

The first stage of study selection involves the screening of titles, abstracts and key words. These will be screened by two independent reviewers for assessment against the inclusion criteria for the review. Potentially relevant studies and other literature will be retrieved in full and imported in the primary screening and data extraction tool Covidence. ${ }^{33}$ The second stage involves a review of the full text of literature identified for inclusion by title, abstract and key word screening. The full text will be assessed in detail against the inclusion criteria by two independent reviewers. Reasons for exclusion of the full text that do not meet the inclusion criteria will be recorded and reported in the scoping review.

\section{Data collection}

Data will be extracted from papers included in the scoping review by two independent reviewers using a charting table (in Covidence) ${ }^{33}$ developed by the reviewers. The data extracted will include specific details about the population, concept, context, study methods (if relevant), level/type of evidence (e.g., peer reviewed, grey literature) and key findings relevant to the review objective. A draft charting table is provided (online supplemental appendix 2). The following details will be extracted from the included literature: author(s), year of publication, type of literature (e.g., research, editorial, report), aim/ purpose, research design (e.g., experimental, quasiexperimental, observational), type of law enforcement officer (e.g., constable, police detachment), context (geographical location and service setting), level of resilience ((1) readiness and preparedness, (2) response and adaptation, (3) recovery and adjustment), ${ }^{22} 26$ characteristics of strategy (guiding principles, main objectives/ outcomes and logistical resources) and main findings/ author's conclusions. Initially, two reviewers will test the charting table on three articles, compare results and make edits if necessary. Following this step, all remaining data extraction will be completed independently by two reviewers and any disagreements that arise between the reviewers will be resolved through discussion or with a third reviewer. If two or more articles describe the same resilience support, it will only be recorded once. Additionally, if data are missing or unclear, authors of the papers will be contacted to request further information. As data extraction continues, the charting table used will likely need adaptation; the final version will be presented in the appendix of the scoping review report.

\section{Data synthesis}

Characteristics of resilience support including guiding principles, objectives/outcomes and logistical resources as well as both the level and source of evidence (e.g., original research report (including research design), government website, editorial) ${ }^{28}$ will be summarised in tabular forms with accompanying text. If other characteristics emerge, they will be presented in the final report. Level of resilience support ((1) readiness and preparedness, (2) response and adaptation, (3) recovery and adjustment) ${ }^{22} 26$ will be summarized thematically, and a narrative explanation with examples (from respective studies/ literature) will also be presented in accordance with the review objective and questions.

\section{Data access}

On completion of the scoping review, data will be made accessible via a data repository located at Dalhousie University, Halifax, Nova Scotia, Canada.

\section{Ethics and dissemination}

The authors do not anticipate any ethical and/or safety considerations occurring from this scoping review and ethics approval is not required. The search for literature will start in November 2020 or upon acceptance of this protocol, whichever comes first. Findings of the scoping review will be available in April 2021. Results from the review will be disseminated in a peer review journal and presented at local, national and international conferences if applicable.

Contributors All authors (AS, CG, AB, CH, ZY and MR) provided substantial contributions to the concept/design of the manuscript; helped draft, provided intellectual content and continual revision; provided final approval of the version to be published and are in agreement/accountable to all aspects of the work submitted. 
Funding The authors have not declared a specific grant for this research from any funding agency in the public, commercial or not-for-profit sectors.

Competing interests None declared.

Patient consent for publication Not required.

Provenance and peer review Not commissioned; externally peer reviewed.

Supplemental material This content has been supplied by the author(s). It has not been vetted by BMJ Publishing Group Limited (BMJ) and may not have been peer-reviewed. Any opinions or recommendations discussed are solely those of the author(s) and are not endorsed by BMJ. BMJ disclaims all liability and responsibility arising from any reliance placed on the content. Where the content includes any translated material, BMJ does not warrant the accuracy and reliability of the translations (including but not limited to local regulations, clinical guidelines, terminology, drug names and drug dosages), and is not responsible for any error and/or omissions arising from translation and adaptation or otherwise.

Open access This is an open access article distributed in accordance with the Creative Commons Attribution Non Commercial (CC BY-NC 4.0) license, which permits others to distribute, remix, adapt, build upon this work non-commercially, and license their derivative works on different terms, provided the original work is properly cited, appropriate credit is given, any changes made indicated, and the use is non-commercial. See: http://creativecommons.org/licenses/by-nc/4.0/.

ORCID iD

Audrey Steenbeek http://orcid.org/0000-0003-0409-158X

\section{REFERENCES}

1 Karaffa KM, Koch JM. Stigma, pluralistic ignorance, and attitudes toward seeking mental health services among police officers. Crim Justice Behav 2016;43:759-77.

2 Blum LN. Force under pressure: how cops live and why they die. 42. New York: Lantern Books, 2000.

3 Papazoglou K, Blumberg DM, Chiongbian VB, et al. The role of moral injury in PTSD among law enforcement officers: a brief report. Front Psychol 2020;11:310 www.frontiersin.org/articles/10.3389/fpsyg. 2020.00310/ful

4 Blum LN. Stoning the keepers at the gate: societies relationship with law enforcement. 17. New York: Lantern Books, 2002.

5 Dempsey JS, Forst LS. An introduction to policing. 149. 3rd ed. Belmont, CA: Thomson Wadsworth, 2015.

6 Soomro S, Yanos PT. Predictors of mental health stigma among police officers: the role of trauma and PTSD. J Police Crim Psych 2019;34:175-83.

7 McCaslin SE, Rogers CE, Metzler TJ, et al. The impact of personal threat on police officers' responses to critical incident stressors. $J$ Nerv Ment Dis 2006;194:591-7.

8 Garbarino S, Guglielmi O, Puntoni M, et al. Sleep quality among police officers: implications and insights from a systematic review and meta-analysis of the literature. Int J Environ Res Public Health 2019;16:885.

9 Bergman AL, Christopher MS, Bowen S. Changes in facets of mindfulness predict stress and anger outcomes for police officers. Mindfulness 2016;7:851-8.

10 Hyungjin K, Jeong L. Organizational stressors associated with six aspects of police officer stress in South Korea. Health Science Journal 2016;10:1-11.

11 Kowalski C. Leadership of first-responders following trauma. J Bus Contin Emer Plan 2019;13:81-90.
12 Van Der Kolk BA. The body keeps the score. Brain, mind, and body in the healing of trauma. NY. Penguin Books 2013;78.

13 Chapin M, Brannen SJ, Singer MI, et al. Training police leadership to recognize and address operational stress. Police Quarterly 2008;11:338-52.

14 Heffren CDJ, Hausdorf PA. Post-Traumatic effects in policing: perceptions, stigmas and help seeking behaviours. Police Practice and Research 2016;17:420-33.

15 Fullerton CS, Ursano RJ, Wang L. Acute stress disorder, posttraumatic stress disorder, and depression in disaster or rescue workers. Am J Psychiatry 2004;161:1370-6.

16 Papazoglou K, Andersen JP. A guide to utilizing police training as a tool to promote resilience and improve health outcomes among police officers. Traumatology 2014;20:103-11.

17 Ungar M. Systemic resilience: principles and processes for a science of change in contexts of adversity. E\&S 2018;23:34.

18 Resilience GNM. paparchy, and world-systems analysis. Ecol Soc 2007;12:24

19 Folke C, Biggs R, Norström AV, et al. Social-ecological resilience and biosphere-based sustainability science. E\&S 2016;21:41.

20 Ungar M. The social ecology of resilience: addressing contextual and cultural ambiguity of a nascent construct. Am J Orthopsychiatry 2011;81:1-17.

21 Masten AS. Resilience in children threatened by extreme adversity: frameworks for research, practice, and translational synergy. Dev Psychopathol 2011;23:493-506.

22 Martin-Breen P, Anderies JM. Resilience: a literature review. Bellagio Initiative 2011:1-67.

23 Janssens KM, van der Velden PG, Taris T, et al. Resilience among police officers: a critical systematic review of used concepts, measures, and predictive values of resilience. J Police Crim Psychol 2018:1-17.

24 Garbarino S, Guglielmi O, Puntoni M, et al. Sleep quality among police officers: implications and insights from a systematic review and meta-analysis of the literature. Int $J$ Environ Res Public Health 2019;16:886.

25 Greinacher A, Derezza-Greeven C, Herzog W, et al. Secondary traumatization in first responders: a systematic review. Eur J Psychotraumatol 2019;10:1562840.

26 Ungar M. Systemic resilience: principles and processes for a science of change in contexts of adversity. Ecology and Society 2018;23:34-50

27 Anglosphere CED. Available: www.collinsdictionary.com/dictionary/ english/anglosphere [Accessed 30 Jun 2020].

28 Centre for Evidence-Based Medicine (CEBM). Available: www.cebm. net/2016/05/ocebm-levels-of-evidence/ [Accessed 30 Jun 2020].

29 Canada S. Police resources in Canada, 2015. Available: https:// www150.statcan.gc.ca/n1/pub/85-002-x/2016001/article/14323eng.htm [Accessed 30 Jun 2020].

30 Bayley $\mathrm{DH}$. The complexities of 21 st century policing. Policing 2016;10:163-70.

31 Peters MDJ, Godfrey C, Mclnerney P, et al. Chapter 11: Scoping reviews. In: Aromataris E, Munn Z, eds. Joanna Briggs Institute Reviewer's Manual [Internet] Adelaide. Joanna Briggs Institute, 2017. https://reviewersmanual.joannabriggs.org/. (accessed 30 Jun 2020).

32 McGowan J, Sampson M, Salzwedel DM, et al. PRESS Peer Review of Electronic Search Strategies: 2015 Guideline Statement. J Clin Epidemiol 2016;75:40-6.

33 Covidence. Available: https://community.cochrane.org/help/toolsand-software/covidence [Accessed July 6, 2020].

34 Tricco AC, Lillie E, Zarin W, et al. PRISMA extension for scoping reviews (PRISMA-ScR): checklist and explanation. Ann Intern Med 2018;169:467. 